\title{
THE STRUCTURE OF THE CRITICAL SET IN THE MOUNTAIN PASS THEOREM
}

\author{
PATRIZIA PUCCI AND JAMES SERRIN
}

\begin{abstract}
We show that the critical set generated by the Mountain Pass Theorem of Ambrosetti and Rabinowitz must have a well-defined structure. In particular, if the underlying Banach space is infinite dimensional then either the critical set contains a saddle point of mountain-pass type, or the set of local minima intersects at least two components of the set of saddle points. Related conclusions are also established for the finite dimensional case, and when other special conditions are assumed. Throughout the paper, no hypotheses of nondegeneracy are required on the critical set.
\end{abstract}

1. Introduction. The existence of critical points of a real-valued $C^{1}$ functional $I$ defined on a real Banach space $X$ has been studied extensively in recent years. Naturally some kind of compactness is required in such problems, the assumption employed here usually being the Palais-Smale condition or some variation of it. A remarkable result in this setting is the following theorem due to Ambrosetti and Rabinowitz [1].

The Mountain Pass Theorem. Let $I: X \rightarrow \mathbf{R}$ be a $C^{1}$ functional satisfying the Palais-Smale condition:

Any sequence $\left(x_{n}\right)$ in $X$ with the property that $I\left(x_{n}\right) \rightarrow$ limit and $I^{\prime}\left(x_{n}\right) \rightarrow 0$ admits a convergent subsequence.

Suppose that there exist two real numbers $a$ and $R, R>0$, such that $I(x) \geq a$ whenever $\|x\|=R, I(0)<a$, and $I(e)<$ a for some $e \in X$ with $\|e\|>R$. Then, with $G$ denoting the class of continuous paths joining 0 and e, the number

$$
b=\inf _{g \in G} \max _{t \in[0,1]} I(g(t))
$$

is a critical value of $I$, i.e. the set

$$
K_{b}=\left\{x \in X: I(x)=b, I^{\prime}(x)=0\right\}
$$

is nonempty.

The structure of the critical set $K_{b}$ has been the subject of recent papers by Hofer and by the present authors. This interest stems from the natural desire to understand the structure of the critical set (e.g. does $K_{b}$ contain a saddle point, as would be expected from its construction), and from the emergence of applications depending on the nature of $K_{b}$ rather than on its mere existence (see [3]).

Received by the editors November 18, 1985.

1980 Mathematics Subject Classification (1985 Revision). Primary 58E05, 49F 15.

Key words and phrases. Critical point theory, nonlinear functional analysis. 
In [4] Hofer showed that the critical set $K_{b}$ possesses either a point of mountainpass type, or a local minimum. On the other hand, in [7] we have shown that when $X$ is infinite dimensional there must be a saddle point in $K_{b}$. Nevertheless the situation still allows considerable refinement, without making any additional hypotheses on the basic functional $I$.

More precisely, the main result of this paper for the infinite dimensional case asserts that either the critical set $K_{b}$ contains a point which is simultaneously of saddle and mountain-pass type, or the set of local minima in $K_{b}$ is nonempty and its closure intersects at least two components of the set of saddle points.

This result can be viewed as a Morse theorem which is applicable to degenerate critical points as well as to functionals merely of class $C^{1}$.

Roughly speaking, the first case of the main result says that the "mountain" surrounding 0 possesses a "realistic" mountain pass. That is, at this point the gradient is zero, in each neighborhood of this point there are higher as well as lower points, and the set of lower points is not path-connected. In other words, the pass must be crossed in going from one set of lower values to another, and at the crossing one can see higher points. Of course, this is not the only conceivable situation, and the second alternative of the theorem gives the other possibility, namely, the mountain pass consists of a saddle point, then of a passage at fixed level through a set of local minima and finally of a second saddle point which is disconnected from the first.

The finite dimensional case, rather remarkably, involves a more subtle discussion. Indeed here, without some additional mild assumptions, the main result no longer holds and must be replaced by a weaker version. More precisely, in this case either the critical set contains a point of mountain-pass type or the set of local minima of $I$ in $K_{b}$ is nonempty and its closure intersects at least two components of the set of saddle points and proper local maxima.

To obtain for the finite dimensional case a result corresponding to that stated earlier for the infinite dimensional case it is necessary to add the hypothesis that the critical set $K_{b}$ does not separate the primary points 0 and $e$ in the Mountain Pass Theorem. That such a hypothesis is not necessary in the infinite dimensional case arises from the underlying strength of the Palais-Smale condition, which implies, among other things, that $K_{b}$ is compact. Since in an infinite dimensional space a compact set cannot separate two different points in its complement, no matter where they may be, there is accordingly no need to invoke the separation hypothesis required for the finite dimensional case.

These results are best possible. Indeed, Rabinowitz has remarked in [8] that, for the finite dimensional case, $K_{b}$ need not contain any saddle point, while in [4] Hofer has given an example where $K_{b}$ contains no points of mountain-pass type. On the other hand, it might be thought that, at least when $K_{b}$ consists of a single point or of isolated points, it should contain a saddle point $y$ not only of mountain-pass type but also having the property that for each sufficiently small neighborhood $N$ of $y$ both sets $\{x \in N: I(x)>b\}$ and $\{x \in N: I(x)<b\}$ are nonempty and not path-connected. Such a generalization, however, is not valid. For example, when $I(x, y, z)=x^{2}+y^{2}+z^{2}(3-z)$ the Mountain Pass Theorem applies with $e=(0,0,3)$ and $a=R=1$. The critical value $b$ is 4 and the critical set $K_{4}$ consists only of the point $(0,0,2)$, which is saddle point of mountain-pass type for which the sets 
$\left\{(x, y, z) \in \mathbf{R}^{3}: x^{2}+y^{2}+(z-2)^{2}<r^{2}\right.$ and $\left.I(x, y, z)>4\right\}, r>0$, are nonempty and path-connected. Similar examples are possible in any finite dimensional space whose dimension $n$ is greater than two and in suitable infinite dimensional spaces.

When $n=2$ and the critical set $K_{b}$ consists of a single point it appears likely that both the sets $\{x \in N: I(x)>b\}$ and $\{x \in N: I(x)<b\}$ are nonempty and not path-connected, but we shall not pursue this.

Precise statements of the results in question, together with their proofs, are given in $\S 3$, following an important preliminary lemma which we establish in $\S 2$. In $\S 4$ we give two further results. The first is essentially a generalization of the theorems of $\S 3$, but with an additional and somewhat special hypothesis. The second concerns the structure of the critical set when $K_{b}$ possibly separates 0 and $e$.

Finally it is worthwhile noting two simple corollaries which follow immediately from the main results.

COROLlARY 1. In the infinite dimensional case, or in the finite dimensional case when the points 0 and $e$ are not separated by the critical set $K_{b}$, there exists a saddle point in $K_{b}$.

Moreover, if the set of saddle points in $K_{b}$ is connected, or if the set of local minima in $K_{b}$, is closed, then there exists a saddle point of mountain-pass type in $K_{b}$.

In particular, if $K_{b}$ contains exactly one saddle point, then this point must be of mountain-pass type.

COROLLARY 2. In the finite dimensional case there exists either a saddle point or a proper local maximum in the critical set $K_{b}$.

In addition, if the set of local minima in $K_{b}$ is closed, then $K_{b}$ contains a point of mountain-pass type.

Finally, if $K_{b}$ contains exactly one point which is either a saddle or a proper local maximum, then this point is of mountain-pass type.

The first part of Corollary 1 was established earlier by the present authors (see Theorem 7 of $[\mathbf{7}]$ ), while Hofer's Theorem given in $[\mathbf{4}]$ is included in Corollaries 1 and 2.

A final remark may be added here. Our principal result shows that the "typical" critical point obtained from the Mountain Pass Theorem is a saddle point of mountain-pass type, as one would expect from the nature of the construction. For illustrative purposes, the functional $I$ may be thought of as having the asymptotic behavior

$$
\sum_{1}^{n-1}\left(x_{i}-x_{i 0}\right)^{2}-\left(x_{n}-x_{n 0}\right)^{2}, \quad X=\mathbf{R}^{n}, n \geq 2,
$$

near such a point. On the other hand, additional types of asymptotic behavior are possible near a saddle point, for example

$$
\sum_{1}^{k-1}\left(x_{i}-x_{i 0}\right)^{2}-\sum_{k}^{n}\left(x_{i}-x_{i 0}\right)^{2}, \quad 3 \leq k \leq n-1, n \geq 4,
$$

this corresponding to a point which is not of mountain-pass type. Clearly, alternative methods are needed to attain such points. 
2. Preliminaries. Hereafter $X$ will be a fixed real Banach space, $I: X \rightarrow \mathbf{R}$ a $C^{1}$ functional satisfying the Palais-Smale condition, and $K_{c}=\{x \in X: I(x)=$ $\left.c, I^{\prime}(x)=0\right\}, c \in \mathbf{R}$, the set of critical points of $I$ at the level $c$.

If $A$ is a subset of $X$ we denote its complement by $A^{c}$, its closure by $\bar{A}$ and its boundary by $\partial A$. If $A$ and $B$ are two nonempty disjoint subsets of $X$ we define the distance from $A$ to $B$ by the number

$$
\operatorname{dist}(A, B)=\inf \{d(x, y): x \in A, y \in B\} .
$$

It is evident that if $A$ is closed and $B$ is compact, for instance, then $0<\operatorname{dist}(A, B)$.

The following lemma is a slight variant of a result due to Clark $[2]$ and Rabinowitz (see the proof of Theorem 1.9 in [8]). For the convenience of the reader we shall give the main outline of the proof.

LEMMA. Let $c$ be a fixed real number and $E$ a nonempty closed set in $X$ such that $E \cap K_{c}=\varnothing$. Then for any positive number $\rho$ and for all sufficiently small $\varepsilon>0$, say $\varepsilon \leq d(c, \rho, E)$, there exists a related continuous mapping $\sigma: X \rightarrow X$ such that:

(i) $\|\sigma(x)-x\| \leq \rho$;

(ii) $I(x) \leq c-2 \varepsilon$ implies $\sigma(x)=x$;

(iii) $x \in E$ and $I(x) \leq c+\varepsilon$ implies $I(\sigma(x)) \leq c-\varepsilon$.

Proof. Assume first that $K_{c} \neq \varnothing$. Then since $K_{c}$ is compact and $E$ closed we have $0<\delta=\operatorname{dist}\left(K_{c}, E\right)$. Put

$$
A_{\delta}=\left\{x \in X: \operatorname{dist}\left(x, K_{c}\right) \geq \delta\right\}
$$

so that $A_{\delta}$ is closed and contains $E$.

By the Palais-Smale condition there exists a positive real number $\alpha$ depending on $\delta$ and having the property that

$$
\left\|I^{\prime}(x)\right\| \geq \alpha>0 \quad \text { when } x \in I^{-1}(c-2, c+2) \cap A_{\delta / 4} .
$$

Given $\rho>0$, we set

$$
d(c, \rho, E)=\min \{1, \alpha \rho / 4, \alpha \delta / 8\},
$$

and consider any positive number $\varepsilon \leq d(c, \rho, E)$.

We now introduce two locally Lipschitz continuous functions $g, \bar{g}: X \rightarrow[0,1]$ such that

$$
g \equiv \begin{cases}0 & \text { outside } I^{-1}(c-2 \varepsilon, c+2 \varepsilon) \\ 1 & \text { in } I^{-1}[c-\varepsilon, c+\varepsilon]\end{cases}
$$

and

$$
\bar{g} \equiv \begin{cases}0 & \text { outside } A_{\delta / 4} \\ 1 & \text { in } A_{\delta / 2}\end{cases}
$$

By Palais' Lemma (for a proof see, for instance, Lemma 1.6 of $[8]$ ) there exists a pseudo-gradient vector field $v: \tilde{X} \rightarrow X, \tilde{X}=\left\{x \in X: I^{\prime}(x) \neq 0\right\}$, of $I$ which is locally Lipschitz continuous. In particular, for $x \in \tilde{X}$

$$
\|v(x)\| \leq 2\left\|I^{\prime}(x)\right\| \quad \text { and } \quad I^{\prime}(x)(v(x)) \geq\left\|I^{\prime}(x)\right\|^{2} .
$$


The mapping $V: X \rightarrow X$ given by

$$
V(x)=-\rho g(x) \bar{g}(x) \frac{v(x)}{\|v(x)\|}
$$

is then well defined. Indeed by (1) and (2) it follows that $\|v(x)\| \geq\left\|I^{\prime}(x)\right\| \geq \alpha>0$ when $x \in I^{-1}(c-2 \varepsilon, c+2 \varepsilon) \cap A_{\delta / 4}$, while $V(x)=0$ when $x \notin I^{-1}(c-2 \varepsilon, c+2 \varepsilon)$ or $x \notin A_{\delta / 4}$ by (3) and (4). By construction $V$ is also bounded and locally Lipschitz continuous. We now consider the flow associated with the ordinary differential equation in $X$

$$
\frac{d \sigma_{x}}{d t}=V\left(\sigma_{x}\right), \quad \sigma_{x}(0)=x
$$

By classical existence theorems there exists a unique solution $\sigma_{x}=\sigma_{x}(t), t \in \mathbf{R}$, for each $x \in X$. Moreover, $\sigma(x) \equiv \sigma_{x}(1)$ is a continuous mapping of $X$ into itself. We shall show that $\sigma$ satisfies properties (i), (ii), and (iii).

Since $\|V(x)\| \leq \rho$ it follows at once that (i) holds. Also in view of (3) we have $\sigma_{x}(t) \equiv x$ whenever $x \notin I^{-1}(c-2 \varepsilon, c+2 \varepsilon)$, proving (ii). It remains to demonstrate (iii).

To this end, put $\omega_{x}(t)=I\left(\sigma_{x}(t)\right)$ and observe that

$$
\begin{aligned}
\frac{d \omega_{x}}{d t}(t) & =I^{\prime}\left(\sigma_{x}(t)\right)\left(\frac{d}{d t} \sigma_{x}(t)\right)=I^{\prime}\left(\sigma_{x}(t)\right)\left(V\left(\sigma_{x}(t)\right)\right) \\
& =-\frac{\rho g\left(\sigma_{x}(t)\right) \bar{g}\left(\sigma_{x}(t)\right)}{\left\|v\left(\sigma_{x}(t)\right)\right\|} I^{\prime}\left(\sigma_{x}(t)\right)\left(v\left(\sigma_{x}(t)\right)\right) \\
& \leq-\frac{\rho g\left(\sigma_{x}(t)\right) \bar{g}\left(\sigma_{x}(t)\right)}{\left\|v\left(\sigma_{x}(t)\right)\right\|}\left\|I^{\prime}\left(\sigma_{x}(t)\right)\right\|^{2} \\
& \leq-\frac{1}{2} \rho g\left(\sigma_{x}(t)\right) \bar{g}\left(\sigma_{x}(t)\right)\left\|I^{\prime}\left(\sigma_{x}(t)\right)\right\|
\end{aligned}
$$

where we have used both properties of the pseudogradient $v$ as well as the fact that $I^{\prime}(x) \neq 0$ when $g(x) \bar{g}(x) \neq 0$. This inequality entails two conclusions: first, for all $x \in X, t \in \mathbf{R}$,

$$
\frac{d \omega_{x}(t)}{d t} \leq 0
$$

and second, for all $x, t$ such that $\sigma_{x}(t) \in I^{-1}[c-\varepsilon, c+\varepsilon] \cap A_{\delta / 2}$,

$$
\frac{d \omega_{x}(t)}{d t} \leq-\frac{1}{2} \rho \alpha
$$

by virtue of (1)-(4).

The first conclusion shows that $I\left(\sigma_{x}(t)\right) \leq c-\varepsilon$ when $I(x) \leq c-\varepsilon$. It is thus enough to obtain (iii) when $x \in I^{-1}(c-\varepsilon, c+\varepsilon] \cap A_{\delta}$.

In this case, suppose for contradiction that $I(\sigma(x))>c-\varepsilon$. Then by (6) we have $\sigma_{x}(t) \in I^{-1}(c-\varepsilon, c+\varepsilon]$ for $t \in[0,1]$. There are now two situations to be considered.

1. $\sigma_{x}(t) \in A_{\delta / 2}$ for all $t \in[0,1]$. Then from (7) and (2) we obtain

a contradiction.

$$
\begin{aligned}
c-\varepsilon & <I(\sigma(x))=\omega_{x}(1)=\omega_{x}(0)+\int_{0}^{1} \frac{d \omega_{x}}{d t}(t) d t \\
& \leq(c+\varepsilon)-\frac{1}{2} \rho \alpha \leq c-\varepsilon
\end{aligned}
$$



find

2. $\sigma_{x}(t) \in A_{\delta / 2}$ for $t \in[0, \tau], \tau<1$, and $\sigma_{x}(\tau) \in \partial A_{\delta / 2}$. Then, as in case 1 , we

$$
c-\varepsilon<I\left(\sigma_{x}(\tau)\right)=\omega_{x}(\tau) \leq(c+\varepsilon)-\frac{1}{2} \rho \alpha \tau,
$$

so that $\rho \tau<4 \varepsilon / \alpha$. On the other hand, since $\|V(x)\|=\rho$ when

$$
x \in I^{-1}[c-\varepsilon, c+\varepsilon] \cap A_{\delta / 2},
$$

we have by $(2)$

$$
\left\|\sigma_{x}(\tau)-\sigma_{x}(0)\right\| \leq \rho \tau<4 \varepsilon / \alpha \leq \delta / 2 .
$$

But $\sigma_{x}(0)=x \in A_{\delta}$ and $\sigma_{x}(\tau) \in \partial A_{\delta / 2}$ which gives $\left\|\sigma_{x}(\tau)-\sigma_{x}(0)\right\| \geq \delta / 2$, violating (8). This completes the demonstration when $K_{c} \neq \varnothing$.

If $K_{c}=\varnothing$ the same proof applies, except that the number $\delta$ need not be introduced, we can simply take $A_{\delta}=A_{\delta / 2}=A_{\delta / 4}=X$, and $\bar{g} \equiv 1$. Moreover the second case above does not now occur since obviously $\sigma_{x}(t) \in X$ for all $t \in \mathbf{R}$.

REMARK. From the proof above it is apparent that for each $x \in X$ there is a continuous map $\sigma_{x}:[0,1] \rightarrow X$ such that $\sigma_{x}(0)=x, \sigma_{x}(1)=\sigma(x)$ and $I\left(\sigma_{x}(t)\right) \leq$ $I(x)$ for every $t \in[0,1]$.

In the remaining part of the paper we shall suppose that $I$ satisfies the hypotheses of the Mountain Pass Theorem, as given in the Introduction. The following notation will be useful throughout the paper:

$$
\begin{aligned}
I_{b}= & \{x \in X: I(x)<b\}, \\
K_{b}= & \left\{x \in X: I(x)=b, I^{\prime}(x)=0\right\}, \\
M_{b}= & \left\{x \in K_{b}: x \text { is a local minimum of } I\right\}, \\
P_{b}=\left\{x \in K_{b}: x \text { is a proper local maximum of } I, \text { that is } x\right. & \text { is a local maximum of } \left.I \text { and } x \in \overline{I_{b}}\right\}, \\
S_{b}=\left\{x \in K_{b}: x \text { is a saddle point of } I,\right. \text { namely in each } & \text { neighborhood of } x \text { there exist two points } y \text { and } z \\
& \text { such that } I(y)<I(x)<I(z)\},
\end{aligned}
$$

where $b$ denotes the critical value of $I$ given in the Mountain Pass Theorem.

It is easily seen that $K_{b}$ is the disjoint union of the sets $M_{b}, P_{b}$ and $S_{b}$. Moreover, in view of the Palais-Smale condition, $K_{b}$ as well as $S_{b}$ and $P_{b} \cup S_{b}$ is compact. Finally $P_{b} \cup S_{b} \subset \overline{I_{b}}$.

We say that $K_{b}$ does not separate 0 and $e$ if there exists a component $C$ of $\left(K_{b}\right)^{c}$ such that $0, e \in C$.

PATHS. A path $g$ joining two points $x_{0}$ and $x_{1}$ in $X$ is by definition a continuous map $g:[0,1] \rightarrow X$ such that $g(0)=x_{0}$ and $g(1)=x_{1}$.

Let $A$ and $B$ be sets in $X$. We call $A$ a subcomponent of $B$ if $A$ is a subset of some component of $B$. In this case, if $B$ is open and $x, y \in A$ then there exists a path joining $x$ and $y$ and contained in $B$.

Now, slightly modifying a terminology introduced by Hofer in [4], we say that a point $x \in K_{b}$ is of mountain-pass type (m-p type) if for any neighborhood $N$ of $x$ the set $N \cap I_{b}$ is not a subcomponent of $I_{b}$. It follows at once that if $x$ is 
of mountain-pass type then for any neighborhood $N$ of $x$ the set $N \cap I_{b}$ must be nonempty and not path-connected (Hofer's definition). Conversely, it is easy to see that if $x$ is not of mountain-pass type then for all suitably small neighborhoods $N$ of $x$ the set $N \cap I_{b}$ must be a subcomponent of $I_{b}$.

We say that a path $g$ intersects a nonempty subset $U$ of $X$ if there exists some $t \in[0,1]$ such that $g(t) \in U$.

Two paths $g$ and $h$ with the property that there exist two parameters $t_{0}$ and $t_{1}$ in $[0,1]$ such that $g\left(t_{0}\right)=h\left(t_{1}\right)$ determine a new path $f$ joining $g(0)$ to $h(1)$ obtained by following, first, $g$ from $g(0)$ to $g\left(t_{0}\right)$ and then $h$ from $h\left(t_{1}\right)$ to $h(1)$. In other words $f:[0,1] \rightarrow X$ is defined by

$$
f(t)= \begin{cases}g\left(2 t_{0} t\right), & 0 \leq t \leq \frac{1}{2} \\ h\left(2\left(1-t_{1}\right) t+2 t_{1}-1\right), & \frac{1}{2} \leq t \leq 1\end{cases}
$$

and clearly is continuous with $f([0,1])=g\left(\left[0, t_{0}\right]\right) \cup h\left(\left[t_{1}, 1\right]\right)$. Obviously the same idea can be used for any finite number of paths having successive intersections.

We finally say that a path $g$ is a bridge if $g([0,1]) \subset I_{b}$. If $x, y$ are two points in a subcomponent of $I_{b}$ then obviously there is a bridge joining these points.

3. The main result. For the sake of simplicity we first prove the main result for the finite dimensional case.

TheOREM 1. Let $X$ be a finite dimensional Banach space. Assume that $K_{b}$ in the Mountain Pass Theorem does not separate 0 and $e$. Then at least one of the following two cases occurs:

$(\alpha) K_{b}$ contains a saddle point of mountain-pass type;

( $\beta) \overline{M_{b}}$ intersects at least two components of $S_{b}$.

ProOF. Without loss of generality we may assume that $X$ is endowed with the Euclidean norm.

Since $K_{b}$ does not separate 0 and $e$, there exists a component $C$ of the open set $\left(K_{b}\right)^{c}$ containing 0 and $e$. Because $C$ is open, it is therefore path-connected as well as connected. Hence there is a path $\hat{g} \in G$ joining 0 and $e$ and lying entirely in $C$. In particular $\hat{g}([0,1]) \cap K_{b}=\varnothing$ and

$$
0<\delta_{1}=\operatorname{dist}\left(\hat{g}([0,1]), K_{b}\right) .
$$

Assume that there are no saddle points in $K_{b}$ of m-p type, for otherwise $(\alpha)$ holds and we are done. Now let us suppose for contradiction that $(\beta)$ fails. Then either

$$
\overline{M_{b}} \cap S_{b}=\varnothing
$$

or

$$
\overline{M_{b}} \text { intersects exactly one component, say } S \text {, of } S_{b} \text {. }
$$

Under the assumption $(*)$ we have

$$
0<\delta_{2}=\operatorname{dist}\left(\overline{M_{b}}, S_{b}\right)
$$

if $M_{b} \neq \varnothing$ and $S_{b} \neq \varnothing$. If either $M_{b}=\varnothing$ or $S_{b}=\varnothing$ then we put $\delta_{2}=1$. The case $S_{b}=\varnothing$ actually does not occur, in view of Theorem 7 of [7], though we make no use of this fact. 
When (**) holds, for each point $x \in S$ we choose an open ball $B\left(x, \rho_{x}\right)$ with center at $x$ and radius $\rho_{x}$ less than $\delta_{1}$ such that $B\left(x, \rho_{x}\right) \cap I_{b}$ is a subcomponent of $I_{b}$, necessarily nonempty since $x$ is a saddle point. This is possible since $(\alpha)$ is assumed false. From the open cover $\left\{B\left(x, \frac{1}{4} \rho_{x}\right): x \in S\right\}$ of the compact set $S$ we can extract a finite subcover, namely

$$
B\left(x_{1}, \frac{1}{4} \rho_{1}\right), \ldots, B\left(x_{k}, \frac{1}{4} \rho_{k}\right), \quad k \geq 1,
$$

whose union will be denoted by $N$. Assumption (**) implies that $N$ is connected, that $\overline{M_{b}}$ and $S_{b} \cap N^{c}$ are disjoint and that

$$
0<\delta_{2}=\operatorname{dist}\left(\overline{M_{b}}, S_{b} \cap N^{c}\right),
$$

if $S_{b} \cap N^{c} \neq \varnothing$. When $S_{b} \cap N^{c}=\varnothing$, we set $\delta_{2}=1$ as before.

For uniformity of notation, we put $N=\varnothing$ and $k=0$ also for the simpler case $(*)$. Let $\delta=\min \left\{\delta_{1}, \delta_{2}\right\}$ and $\hat{K}_{b}=K_{b} \cap N^{c}$.

We now introduce an open cover for $\hat{K}_{b}$. Since none of the saddle points $x$ of $\hat{K}_{b}$ is of m-p type, for each of them we again select an open ball $B\left(x, \rho_{x}\right)$ with center at $x$ and radius $\rho_{x}<\delta$ such that $B\left(x, \rho_{x}\right) \cap I_{b}$ is a subcomponent of $I_{b}$. If $y \in \hat{K}_{b}$ is a local minimum of $I$, we choose an open ball $B\left(y, r_{y}\right)$ such that $I \geq b$ in $B\left(y, r_{y}\right)$. Similarly, if $y \in \hat{K}_{b}$ is a proper local maximum of $I$, we pick an open ball $B\left(y, r_{y}\right)$ such that $I \leq b$ in $B\left(y, r_{y}\right)$. Again in these two last cases we take the radii $r_{y}<\delta$. The open cover $\left\{B\left(x, \frac{1}{4} \rho_{x}\right), B\left(y, \frac{1}{2} r_{y}\right): x \in \hat{K}_{b} \cap S_{b}, y \in \hat{K}_{b} \cap S_{b}^{c}\right\}$ of the compact set $\hat{K}_{b}$ has a finite subcover of the form:

$$
\begin{gathered}
B\left(x_{k+1}, \frac{1}{4} \rho_{k+1}\right), \ldots, B\left(x_{k+l}, \frac{1}{4} \rho_{k+l}\right), \\
B\left(y_{1}, \frac{1}{2} r_{1}\right), \ldots, B\left(y_{m}, \frac{1}{2} r_{m}\right), \\
B\left(y_{m+1}, \frac{1}{2} r_{m+1}\right), \ldots, B\left(y_{m+n}, \frac{1}{2} r_{m+n}\right),
\end{gathered}
$$

whose union will be denoted by $W$. Here $x_{k+1}, \ldots, x_{k+l}$ are saddle points of $I$; $y_{1}, \ldots, y_{m}$ are local minima of $I$; and $y_{m+1}, \ldots, y_{m+n}$ are proper local maxima of $I$ in $\hat{K}_{b}$. If a ball $B\left(y_{i}, \frac{1}{2} r_{i}\right)$ arises from a flat point $y_{i}$ of $I$, it will of course appear only in (11). Possibly there are no balls of any particular type (9)-(12), but this will not affect the proof. In the following $U$ will denote the open neighborhood $N \cup W$ of $K_{b}$. Clearly $U \cap \hat{g}([0,1])=\varnothing$.

Since $U$ is given by a finite union of open balls, it can be assumed that its components $U_{j}, j=1, \ldots, \mu, \mu \leq 1+l+m+n$, which are of course open, have the additional property that

$$
0<\min \left\{\operatorname{dist}\left(\overline{U_{i}}, \overline{U_{j}}\right): i, j=1, \ldots, \mu, i \neq j\right\} .
$$

In fact, if necessary, we may attain this requirement by slightly reducing each of the numbers $\rho_{i}, i=k+1, \ldots, k+l$, and $r_{s}, s=1, \ldots, m+n$.

Since $K_{b}$ is compact and $U$ is an open neighborhood of $K_{b}$ it is clear that

$$
0<\rho=\frac{1}{2} \operatorname{dist}\left(K_{b}, U^{c}\right) \text {. }
$$

Define $E=\left\{x \in X: \operatorname{dist}\left(x, U^{c}\right) \leq \rho\right\}$, so that $E$ is closed, disjoint from $K_{b}$ and contains $U^{c}$. Let $d=d(b, \rho, E)$ be the number given in the lemma of $\S 2$ when $K_{c}=K_{b}$, and put

$$
\varepsilon=\min \left\{d, \frac{1}{2}(b-I(0)), \frac{1}{2}(b-I(e))\right\} .
$$


Clearly $\varepsilon>0$ since $b \geq a>\max \{I(0), I(e)\}$. In view of the definition of $b$ we can fix a path $g$ such that

$$
g \in G, \quad g([0,1]) \subset I_{b+\varepsilon} .
$$

Our goal is to modify $g$ to obtain another path $\tilde{g}$ such that

$$
\tilde{g} \in G, \quad \tilde{g}([0,1]) \subset I_{b} .
$$

This will contradict the definition of $b$ and complete the proof.

Consider the mapping $\sigma: X \rightarrow X$ given by the lemma in $\S 2$, depending on $b, \rho, E$ and $\varepsilon$. By the definition of $\varepsilon$ and property (ii) in the lemma it is evident that the path $\sigma \circ g$ is in $G$. Moreover for all $t$ such that $\sigma \circ g(t) \in U^{c}$ we have $I(\sigma \circ g(t)) \leq b-\varepsilon$. Indeed the condition $\sigma \circ g(t) \in U^{c}$ together with property (i) of the lemma shows that $g(t) \in E$. But then $I(\sigma \circ g(t)) \leq b-\varepsilon$ in view of (13), property (iii) of the lemma, and the fact that $\varepsilon \leq d$.

We may assume without loss of generality, therefore, that the path $g$ itself satisfies the condition

$$
g(t) \in U^{c} \quad \text { implies } \quad I(g(t)) \leq b-\varepsilon,
$$

and a fortiori that

$$
g(t) \in U^{c} \quad \text { implies } g(t) \in I_{b} .
$$

If $g([0,1]) \subset U^{c}$, then we reach the required contradiction immediately. Thus, it is enough to consider the case when $g$ intersects $U$. We can suppose that the first component $U_{j}$ of $U$ intersected by $g$, in the natural order given by $t$, is $U_{1}$. Let $t_{1}^{0}$, $t_{1}^{1}$ be the unique pair of parameters such that

$$
\begin{aligned}
& 0<t_{1}^{0}<t_{1}^{1}<1 \\
& z_{1}^{0}=g\left(t_{1}^{0}\right), z_{1}^{1}=g\left(t_{1}^{1}\right) \in \partial U_{1} \\
& g\left(\left[0, t_{1}^{0}\right]\right) \cap U=g\left(\left[t_{1}^{1}, 1\right]\right) \cap U_{1}=\varnothing .
\end{aligned}
$$

In the same manner, the first component $U_{j}$ intersected by $g$ for some $t>t_{1}^{1}$ can be supposed to be $U_{2}$. Again there exists a unique pair of parameters $t_{2}^{0}, t_{2}^{1}$ such that

$$
\begin{aligned}
& t_{1}^{1}<t_{2}^{0}<t_{2}^{1}<1 \\
& z_{2}^{0}=g\left(t_{2}^{0}\right), z_{2}^{1}=g\left(t_{2}^{1}\right) \in \partial U_{2} \\
& g\left(\left[t_{1}^{1}, t_{2}^{0}\right]\right) \cap U=g\left(\left[t_{2}^{1}, 1\right]\right) \cap U_{2}=\varnothing .
\end{aligned}
$$

Now, proceeding recursively, we construct $\nu$ pairs of parameters, with $\nu \leq \mu$, such that

$$
\begin{gathered}
0=t_{0}^{1}<t_{1}^{0}<t_{1}^{1}<\cdots<t_{\nu}^{0}<t_{\nu}^{1}<t_{\nu+1}^{0}=1 \\
z_{j}^{0}=g\left(t_{j}^{0}\right), z_{j}^{1}=g\left(t_{j}^{1}\right) \in \partial U_{j}, \quad j=1, \ldots, \nu \\
\bigcup_{j=1}^{\nu+1} g\left(\left[t_{j-1}^{1}, t_{j}^{0}\right]\right) \subset I_{b} .
\end{gathered}
$$

For uniformity of notation we write $0=z_{0}^{1}$ and $e=z_{\nu+1}^{0}$. Note also that each of the points $z_{j}, j=1, \ldots, \nu$, is in $I_{b}$, by the result of the previous paragraph. 
We now show that there exists a bridge $v_{j}$ corresponding to each pair of points $z_{j}^{0}$ and $z_{j}^{1}, j=1, \ldots, \nu$. We begin by constructing the bridge $v_{1}$ from $z_{1}^{0}$ to $z_{1}^{1}$.

Since by assumption $K_{b}$ does not separate 0 and $e$, we claim that $z_{1}^{0}$ and $z_{1}^{1}$ are in the same component $L_{1}$ of $\left(U_{1}\right)^{c}$. To show this, we first follow the path $g$ from $z_{1}^{1}$ until we reach $e$. Afterwards we proceed along the continuous path $\hat{g}$ in the reverse direction from $e$ to 0 , and then along $g$ from 0 to $z_{1}^{0}$. The resulting continuous path joins $z_{1}^{1}$ to $z_{1}^{0}$ and nowhere intersects $U_{1}$. This proves the claim above.

At the same time, $z_{1}^{0}, z_{1}^{1}$ are in $\partial U_{1}$ and hence also belong to $\partial L_{1}$. By the lemma in $\S 7$ of $[7]$ we know that $\partial L_{1}$ is path-connected. Thus, there exists a continuous path $h_{1}$ joining $z_{1}^{0}$ and $z_{1}^{1}$ with $h_{1}([0,1]) \subset \partial L_{1}$. We recall that $h_{1}(0)=z_{1}^{0}$ and $h_{1}(1)=z_{1}^{1}$ are both in $I_{b}$. If $h_{1}([0,1]) \subset I_{b}$, then the path $v_{1}=h_{1}$ is a bridge from $z_{1}^{0}$ to $z_{1}^{1}$.

The converse case, $h_{1}([0,1]) \not \subset I_{b}$, requires a further construction. Let us write

$$
\partial U=\Gamma_{1} \cup \Gamma_{2} \cup \Gamma_{3}
$$

where

$$
\begin{aligned}
& \Gamma_{1}=\bigcup_{i=1}^{k+l}\left(\partial U \cap \partial B\left(x_{i}, \frac{1}{4} \rho_{i}\right)\right), \\
& \Gamma_{2}=\bigcup_{s=1}^{m}\left(\partial U \cap \partial B\left(y_{s}, \frac{1}{2} r_{s}\right)\right), \\
& \Gamma_{3}=\bigcup_{s=m+1}^{m+n}\left(\partial U \cap \partial B\left(y_{s}, \frac{1}{2} r_{s}\right)\right) .
\end{aligned}
$$

In the following, to simplify the notation, we shall denote the closed balls $\overline{B\left(x_{i}, \frac{1}{4} \rho_{i}\right)}$ by $B_{i}$ and the sets $B\left(x_{i}, \rho_{i}\right) \cap I_{b}$ by $I_{b}^{i}$. For later use, recall that each of the sets $I_{b}^{i}$ is a nonempty subcomponent of $I_{b}$. It will be convenient also to refer to the closure of any ball listed in (9) as a closed ball of type (9), and similarly for the closures of balls listed in (10), (11), and (12).

If $x \in \Gamma_{3}$, then $x \in \partial B\left(y_{s}, \frac{1}{2} r_{s}\right)$ for some $s=m+1, \ldots, m+n$. Since $I \leq b$ in $B\left(y_{s}, r_{s}\right)$, the equality $I(x)=b$ implies $I^{\prime}(x)=0$. But this is impossible since $x \notin K_{b}$. Hence $I<b$ in $\Gamma_{3}$, and by the compactness of $\Gamma_{3}$ there exists a real number $c$ such that $I \leq c<b$ in $\Gamma_{3}$. Moreover $\Gamma_{2} \cap \Gamma_{3}=\varnothing$ and $\Gamma_{2} \cap I_{b}=\varnothing$, since $I \geq b$ in $\Gamma_{2}$.

We select a parameter $\tau_{1}^{0} \in[0,1)$ as follows:

(a) if $I\left(h_{1}(0)\right)<\frac{1}{2}(b+c)$, then $\tau_{1}^{0}$ is the maximal parameter in $(0,1)$ such that $I\left(h_{1}\left(\tau_{1}^{0}\right)\right)=\frac{1}{2}(b+c)$ and $I\left(h_{1}(\tau)\right) \leq \frac{1}{2}(b+c)$ for $\tau \in\left[0, \tau_{1}^{0}\right]$;

(b) if $I\left(h_{1}(0)\right) \geq \frac{1}{2}(b+c)$, then $\tau_{1}^{0}=0$.

The existence of $\tau_{1}^{0}$ in (a) follows from the present assumption that $h_{1}([0,1]) \not \subset I_{b}$. Here and in the following the parameters $\tau$ which we introduce depend on the path $h_{1}$, but for simplicity we do not indicate this dependence explicitly. In both cases (a) and (b) we have $c<I\left(h_{1}\left(\tau_{1}^{0}\right)\right)<b$, and so $h_{1}\left(\tau_{1}^{0}\right) \in \Gamma_{1}$.

Let $C_{1}$ be the component of the compact set $\Gamma_{1} \cup \Gamma_{2}$ containing $h_{1}\left(\tau_{1}^{0}\right)$ and $\tau_{1}^{1}$ the maximum value of $\tau$ such that $h_{1}(\tau) \in C_{1}$. If $\tau_{1}^{1}<1$, then $h_{1}\left(\tau_{1}^{1}\right) \in \Gamma_{3}$. Otherwise, there exists $\theta>0$ such that $h_{1}\left(\left[\tau_{1}^{1}-\theta, \tau_{1}^{1}+\theta\right]\right) \subset \partial U \backslash \Gamma_{3} \subset \Gamma_{1} \cup \Gamma_{2}$. Hence, since $C_{1}$ is a component of $\Gamma_{1} \cup \Gamma_{2}$ and $h_{1}\left(\tau_{1}^{1}\right) \in C_{1}$, we get $h_{1}\left(\tau_{1}+\theta\right) \in C_{1}$, which 
is absurd. Thus, if $\tau_{1}^{1}<1$, then $h_{1}\left(\tau_{1}^{1}\right) \in\left(\Gamma_{1} \cup \Gamma_{2}\right) \cap \Gamma_{3} \subset \Gamma_{1} \cap I_{b}$. Moreover, since $h_{1}\left(\tau_{1}^{1}\right) \in \Gamma_{3}$ we have $I\left(h_{1}\left(\tau_{1}^{1}\right)\right) \leq c$, while on the other hand $I\left(h_{1}\left(\tau_{1}^{0}\right)\right) \geq$ $\frac{1}{2}(b+c)>c$. Consequently $\tau_{1}^{1}>\tau_{1}^{0}$. If $\tau_{1}^{1}=1$, then by construction we again have $h_{1}\left(\tau_{1}^{1}\right)=h_{1}(1)=z_{1}^{1} \in C_{1} \cap I_{b} \subset \Gamma_{1} \cap I_{b}$. Finally we observe that $h_{1}\left(\tau_{1}^{0}\right)$, $h_{1}\left(\tau_{1}^{1}\right) \in C_{1} \cap \Gamma_{1} \cap I_{b}$ and hence in particular $C_{1} \cap \Gamma_{1} \neq \varnothing$.

The next step is to show that there exists a subcomponent $I_{b}^{(1)}$ of $I_{b}$ containing both $h_{1}\left(\tau_{1}^{0}\right)$ and $h_{1}\left(\tau_{1}^{1}\right)$. It is convenient to discuss assumptions $(*)$ and $(* *)$ separately.

Assumption (*). We recall that here $k=0$. If $x \in \Gamma_{1} \cap \Gamma_{2}$, then there exist two indices $i$ and $s, i=1, \ldots, l, s=1, \ldots, m$, such that $\left\|x-x_{i}\right\|=\frac{1}{4} \rho_{i}$ and $\left\|x-y_{s}\right\|=$ $\frac{1}{2} r_{s}$. Therefore $\left\|y_{s}-x_{i}\right\| \leq \frac{1}{2} r_{s}+\frac{1}{4} \rho_{i}<\delta \leq \delta_{2}=\operatorname{dist}\left(\overline{M_{b}}, S_{b}\right)$, which is absurd since $y_{s} \in M_{b}$ and $x_{i} \in S_{b}$. Thus $\Gamma_{1} \cap \Gamma_{2}=\varnothing$. Since $C_{1}=\left(C_{1} \cap \Gamma_{1}\right) \cup\left(C_{1} \cap \Gamma_{2}\right)$ and $C_{1}$ is connected, it now follows that $C_{1} \cap \Gamma_{2}=\varnothing$ and hence $C_{1} \subset \Gamma_{1}$. Consequently there exist $l^{\prime}$ saddle points, $l^{\prime} \leq l$, which without loss of generality we may relabel $x_{1}, x_{2}, \ldots, x_{l^{\prime}}$, such that $\bigcup_{j=1}^{l^{\prime}} \partial B_{j}$ is connected and contains $C_{1}$. In particular, denoting the set $\bigcup_{j=1}^{l^{\prime}} I_{b}^{j}$ by $I_{b}^{(1)}$, we have $h_{1}\left(\tau_{1}^{0}\right), h_{1}\left(\tau_{1}^{1}\right) \in C_{1} \cap I_{b} \subset I_{b}^{(1)}$.

We now show that $I_{b}^{(1)}$ is a subcomponent of $I_{b}$. To see this, consider two balls $B_{i}, B_{j}, i, j=1, \ldots, l^{\prime}, i \neq j$, such that $B_{i} \cap B_{j} \neq \varnothing$. Then, either $x_{j} \in B\left(x_{i}, \rho_{i}\right)$ or $x_{i} \in B\left(x_{j}, \rho_{j}\right)$. In both cases $I_{b}^{i} \cap I_{b}^{j} \neq \varnothing$ and therefore $I_{b}^{i} \cup I_{b}^{j}$ is a subcomponent of $I_{b}$. Next let $x, y$ be two points in $I_{b}^{(1)}$, say $x \in B\left(x_{1}, \rho_{1}\right)$ and $y \in B\left(x_{l^{\prime}}, \rho_{l^{\prime}}\right)$. Since $\bigcup_{j=1}^{l^{\prime}} B_{j}$ is connected, a standard maximality argument shows that there exists a chain of the balls $B_{j_{s}}$ in this union joining $B_{1}$ and $B_{l^{\prime}}$, namely $B_{j_{1}}=B_{1}, B_{l_{l^{\prime \prime}}}=B_{l^{\prime}}$, $B_{j_{s}} \cap B_{j_{s+1}} \neq \varnothing, s=1, \ldots, l^{\prime \prime}-1, l^{\prime \prime} \leq l^{\prime}$. Hence $I_{b}^{(1)}$ is a subcomponent of $I_{b}$, as desired.

Assumption (**). Let $\hat{N}$ be the component of the set $\bigcup_{i=1}^{k+l} B_{i}$ which contains the open connected set $N$. Since $h_{1}\left(\tau_{1}^{0}\right), h_{1}\left(\tau_{1}^{1}\right) \in C_{1} \cap \Gamma_{1} \cap I_{b}$ it follows that $h_{1}\left(\tau_{1}^{0}\right), h_{1}\left(\tau_{1}^{1}\right) \in \bigcup_{i=1}^{k+l} B_{i}$. There are now two cases to be discussed, that is $\left\{h_{1}\left(\tau_{1}^{0}\right), h_{1}\left(\tau_{1}^{1}\right)\right\} \subset \hat{N}$ and $\left\{h_{1}\left(\tau_{1}^{0}\right), h_{1}\left(\tau_{1}^{1}\right)\right\} \not \subset \hat{N}$. If the first case occurs, then $h_{1}\left(\tau_{1}^{0}\right), h_{1}\left(\tau_{1}^{1}\right) \in \hat{N} \cap I_{b}$. This set, which we again call $I_{b}^{(1)}$, is a subcomponent of $I_{b}$ by the argument of the previous paragraph.

Next suppose $\left\{h_{1}\left(\tau_{1}^{0}\right), h_{1}\left(\tau_{1}^{1}\right)\right\} \not \subset \hat{N}$, say, for instance, $h_{1}\left(\tau_{1}^{0}\right) \notin \hat{N}$. We shall show then that $C_{1} \subset \Gamma_{1}$, so that the situation reduces to the case discussed in (*). Thus assume for contradiction that $C_{1} \cap \Gamma_{2} \neq \varnothing$. Hence there exists some index $\tilde{s}=1, \ldots, m$ such that $C_{1} \cap \partial B\left(y_{\tilde{s}}, \frac{1}{2} r_{\tilde{s}}\right) \neq \varnothing$. Consequently, since $C_{1}$ is connected, there must be a chain joining $h_{1}\left(\tau_{1}^{0}\right)$ and $\overline{B\left(y_{\tilde{s}}, \frac{1}{2} r_{\tilde{s}}\right)}$ consisting of closed balls of type (9) or (10) or (11). The first ball in the chain must be of type (9) or (10) since $h_{1}\left(\tau_{1}^{0}\right) \in I_{b}$. Hence without loss of generality we may assume that all balls $\tilde{B}$ in the chain before $\overline{B\left(y_{\tilde{s}}, \frac{1}{2} r_{\tilde{s}}\right)}$ are of type (9) or (10). By the definition of $\hat{N}$ and the fact that $h_{1}\left(\tau_{1}^{0}\right) \notin \hat{N}$, it now follows that the balls $\tilde{B}$ cannot intersect $\hat{N}$ and so a fortiori cannot intersect $N$. In particular, each ball $\tilde{B}$ is of type (10). But this cannot occur since closed balls of type (10) do not intersect closed balls of type (11), by construction and the fact that $\delta \leq \delta_{2}$. Thus, $C_{1} \subset \Gamma_{1}$ as required.

The treatment of the case $h_{1}\left(\tau_{1}^{1}\right) \notin \hat{N}$ is exactly the same. 
For both assumptions $(*)$ and $(* *)$ we stop the construction if $\tau_{1}^{1}=1$. Otherwise, recalling that $I\left(h_{1}\left(\tau_{1}^{1}\right)\right)<\frac{1}{2}(b+c)$ and repeating the procedure as often as necessary, we ultimately obtain a finite set of parameters $\tau$ such that

$$
\begin{gathered}
0=\tau_{0}^{1} \leq \tau_{1}^{0}<\tau_{1}^{1}<\cdots<\tau_{\lambda}^{0}<\tau_{\lambda}^{1} \leq \tau_{\lambda+1}^{0}=1 \\
h_{1}\left(\tau_{s}^{0}\right), h_{1}\left(\tau_{s}^{1}\right) \in I_{b}^{(s)} \quad \text { for every } s=1, \ldots, \lambda \\
\bigcup_{s=1}^{\lambda+1} h_{1}\left(\left[\tau_{s-1}^{1}, \tau_{s}^{0}\right]\right) \subset I_{b}
\end{gathered}
$$

where the set $I_{b}^{(s)}$ is a subcomponent of $I_{b}$ for every $s=1, \ldots, \lambda$. That the parameter set (18) is not infinite follows from the fact that the continuous function $I \circ h_{1}$ on $[0,1]$ cannot have an infinite number of oscillations between the two values $c$ and $\frac{1}{2}(b+c)$. Now by $(19)$ the points $h_{1}\left(\tau_{s}^{0}\right)$ and $h_{1}\left(\tau_{s}^{1}\right)$ are in the same subcomponent of $I_{b}$, so there exists a bridge $v_{s}^{1}$ joining them. We are thus in position to construct a bridge $v_{1}$ from $z_{1}^{0}$ to $z_{1}^{1}$. Indeed, it is enough to follow the path $h_{1}$ from $z_{1}^{0}=h_{1}(0)$ to $h_{1}\left(\tau_{1}^{0}\right)$, next $v_{1}^{1}$ from $h_{1}\left(\tau_{1}^{0}\right)$ to $h_{1}\left(\tau_{1}^{1}\right)$, subsequently $h_{1}$ from $h_{1}\left(\tau_{1}^{1}\right)$ to $h_{1}\left(\tau_{2}^{0}\right)$ and so forth until we reach $z_{1}^{1}=h_{1}(1)$.

Proceeding in this way we can therefore successively bridge each of the components $U_{j}, j=1, \ldots, \nu$, by a corresponding path $v_{j}$ from $z_{j}^{0}$ to $z_{j}^{1}$.

We can now obtain the required path $\tilde{g}$ satisfying (14). In fact it is sufficient to follow $g$ from $0=z_{0}^{1}$ to $z_{1}^{0}$, then $v_{1}$ from $z_{1}^{0}$ to $z_{1}^{1}$, next $g$ from $z_{1}^{1}$ to $z_{2}^{0}$ and so on until reaching $e=z_{\nu+1}^{0}$. This completes the proof.

Now we state the main result for the infinite dimensional case:

THEOREM 2. Let $X$ be an infinite dimensional Banach space. Then at least one of the following two cases occurs:

$(\alpha) K_{b}$ contains a saddle point of mountain-pass type;

( $\beta) \overline{M_{b}}$ intersects at least two components of $S_{b}$.

ProOF. Since $X$ is infinite dimensional and 0 and $e$ do not belong to the compact set $K_{b}$, they both are in the same component $C$ of $\left(K_{b}\right)^{c}$. Thus we may follow the main argument introduced for the finite dimensional case. For this reason, there will be no confusion if we do not repeat word for word the previous preliminary construction. Indeed, no changes are necessary in the selection of the number $\delta=\min \left\{\delta_{1}, \delta_{2}\right\}$ and of the open neighborhood $U=N \cup W$ of $K_{b}$. It is also worth noting here that $K_{b}$ contains no flat points. We introduce as before

$$
\rho=\frac{1}{2} \operatorname{dist}\left(K_{b}, U^{c}\right), \quad E=\left\{x \in X: \operatorname{dist}\left(x, U^{c}\right) \leq \rho\right\}, \quad d=d(b, \rho, E),
$$

and take

$$
\varepsilon=\min \left\{d, \frac{1}{2}(b-I(0)), \frac{1}{2}(b-I(e))\right\} .
$$

We now fix a path $g$ satisfying (13) and shall show that it can be modified in order to obtain (14). To see this, we proceed word for word until formula (17).

Thus, it remains to show that there exists a bridge $v_{j}$ corresponding to each pair of points $z_{j}^{0}, z_{j}^{1}, j=1, \ldots, \nu$. To begin with, we modify the path $\hat{g}$ joining 0 and $e$ into a piecewise linear path, still from 0 to $e$ and nowhere intersecting $\bar{U}$, which we continue to call $\hat{g}$ for simplicity. Then we approximate the parts of $g$ joining $z_{j}^{1}$ 
and $z_{j+1}^{0}$ by piecewise linear paths $g_{j}$ from $z_{j}^{1}$ to $z_{j+1}^{0}$, entirely contained in $I_{b}$ for each $j=0,1, \ldots, \nu$.

Let $Y$ be a finite dimensional subspace of $X$ containing the piecewise linear paths $\hat{g}([0,1])$ and $g_{j}([0,1]), j=0,1, \ldots, \nu$, as well as the centers of the set of balls listed in (9)-(12). Without loss of generality we may endow $Y$ with the Euclidean norm.

Let $U^{\prime}=U \cap Y, N^{\prime}=N \cap Y$ and $U_{j}^{\prime}=U_{j} \cap Y$, for $j=1, \ldots, \mu$. Clearly $N^{\prime}$ is connected and $U_{j}^{\prime}$ are exactly the components of $U^{\prime}$ in $Y$.

For each point $x \in \overline{U^{\prime}}$ we choose an open ball $B_{Y}\left(x, r_{x}\right)$ in $Y$ such that:

$$
B_{Y}\left(x, r_{x}\right) \cap \hat{g}([0,1])=\varnothing ;
$$

if $x$ belongs to some closed ball of type (9)-(12), then $B_{Y}\left(x, r_{x}\right)$ is entirely contained in the corresponding ball of radius $\frac{1}{3} \rho_{i}, i=$ $1, \ldots, k+l$, or $\frac{2}{3} r_{s}, s=1, \ldots, m+n$

if $x$ and $\tilde{x}$ respectively are in disjoint closed balls of type (9)-(12), then the closures of $B_{Y}\left(x, r_{x}\right)$ and $B_{Y}\left(\tilde{x}, r_{\tilde{x}}\right)$ are disjoint.

From the open cover $\left\{B_{Y}\left(x, r_{x}\right): x \in \overline{U^{\prime}}\right\}$ of the compact set $\overline{U^{\prime}}$ of $Y$ we select a finite subcover, whose union will be called $V$. We denote by $V_{j}$ the component of $V$ containing $U_{j}^{\prime}, j=1, \ldots, \mu$, so that by (23) we have $\overline{V_{i}} \cap \overline{V_{j}}=\varnothing$ if $i \neq j$.

For uniformity and simplicity of notation we still call $z_{j}^{0}$ the first point of intersection of $g_{j-1}$ with $\partial V_{j}$ and $z_{j}^{1}$ the last point of intersection of $g_{j}$ with $\partial V_{j}$, $j=1, \ldots, \mu$. The argument already used for the finite dimensional case implies that $z_{j}^{0}, z_{j}^{1}$ are in the same component $L_{j}$ of $Y \backslash V_{j}$. Hence, by the lemma in $\S 7$ of [7], there is a path $h_{j}$ from $z_{j}^{0}$ to $z_{j}^{1}$ entirely contained in $\partial L_{j}$ for each $j=1, \ldots, \mu$.

We start by constructing a bridge $v_{1}$ joining $z_{1}^{0}$ to $z_{1}^{1}$. If $h_{1}([0,1]) \subset I_{b}$, we take $v_{1}=h_{1}$ as before. Otherwise we introduce the following new sets:

$$
\begin{aligned}
& \Gamma_{1}^{\prime}=\partial V \cap \bigcup_{i=1}^{k+l} \overline{B\left(x_{i}, \frac{1}{3} \rho_{i}\right)}, \\
& \Gamma_{2}^{\prime}=\partial V \cap \bigcup_{s=1}^{m} \overline{B\left(y_{s}, \frac{2}{3} r_{s}\right)}, \\
& \Gamma_{3}^{\prime}=\partial V \cap \bigcup_{s=m+1}^{l} \overline{B\left(y_{s}, \frac{2}{3} r_{s}\right)}
\end{aligned}
$$

By (22) it is clear that $\partial V=\Gamma_{1}^{\prime} \cup \Gamma_{2}^{\prime} \cup \Gamma_{3}^{\prime}$. Moreover as in the proof of Theorem 1 we have $I(z)<b$ on the closed bounded subset $\Gamma_{3}^{\prime}$ of $Y$, so in turn there exists a real number $c$ such that $I \leq c<b$ in $\Gamma_{3}^{\prime}$. Since $I \geq b$ on $\Gamma_{2}^{\prime}$ it follows also that $\Gamma_{2}^{\prime} \cap I_{b}=\varnothing$ and $\Gamma_{2}^{\prime} \cap \Gamma_{3}^{\prime}=\varnothing$.

Define $\tau_{1}^{0} \in[0,1)$ as in the proof of Theorem 1 . As before, $h_{1}\left(\tau_{1}^{0}\right)$ is in $\Gamma_{1}^{\prime}$ but not in $\Gamma_{2}^{\prime}$ or $\Gamma_{3}^{\prime}$. Let $C_{1}$ be the component of the compact set $\Gamma_{1}^{\prime} \cup \Gamma_{2}^{\prime}$ containing $h_{1}\left(\tau_{1}^{0}\right)$ and $\tau_{1}^{1}$ the maximum value of $\tau$ such that $h_{1}(\tau) \in C_{1}$. Arguing as in the finite dimensional case, we see at once that $h_{1}\left(\tau_{1}^{1}\right) \in \Gamma_{3}^{\prime}$ when $\tau_{1}^{1}<1$. In this case $h_{1}\left(\tau_{1}^{1}\right) \in\left(\Gamma_{1}^{\prime} \cup \Gamma_{2}^{\prime}\right) \cap \Gamma_{3}^{\prime} \subset \Gamma_{1}^{\prime} \cap I_{b}$. Also as before $\tau_{1}^{0}<\tau_{1}^{1}$. When $\tau_{1}^{1}=1$, the 
definition of $\tau_{1}^{1}$ shows that $h_{1}\left(\tau_{1}^{1}\right)=h_{1}(1)=z_{1}^{1} \in C_{1} \cap I_{b} \subset \Gamma_{1}^{\prime} \cap I_{b}$. In both cases $h_{1}\left(\tau_{1}^{0}\right), h_{1}\left(\tau_{1}^{1}\right) \in C_{1} \cap \Gamma_{1}^{\prime} \cap I_{b}$ and so in particular $C_{1} \cap \Gamma_{1}^{\prime}$ is nonempty. $(* *)$.

For convenience at this stage we separate the treatment of assumptions $(*)$ and

Assumption (*). We recall that $k=0$ here. Moreover $\Gamma_{1}^{\prime} \cap \Gamma_{2}^{\prime}=\varnothing$, for if not then there must exist a point $y$ such that $y \in \overline{B\left(x_{i}, \frac{1}{3} \rho_{i}\right)}$ for some $i=1, \ldots, l$ and $y \in \overline{B\left(y_{s}, \frac{2}{3} r_{s}\right)}$ for some $s=1, \ldots, m$. Hence $\left\|y_{s}-x_{i}\right\|<\delta \leq \delta_{2}=\operatorname{dist}\left(\overline{M_{b}}, S_{b}\right)$, which is absurd. Thus we have $C_{1} \cap \Gamma_{2}^{\prime}=\varnothing$ by the usual connectedness argument, and so $C_{1} \subset \Gamma_{1}^{\prime}$. Therefore there exist $l^{\prime}$ saddle points, $l^{\prime} \leq l$, which we may still call $x_{1}, x_{2}, \ldots, x_{l^{\prime}}$, such that $\bigcup_{j=1}^{l^{\prime}} \overline{B\left(x_{j}, \frac{1}{3} \rho_{j}\right)}$ is connected and contains $C_{1}$. We also have $h_{1}\left(\tau_{1}^{0}\right), h_{1}\left(\tau_{1}^{1}\right) \in C_{1} \cap I_{b} \subset I_{b}^{(1)}$ where, as usual, $I_{b}^{(1)}=\bigcup_{j=1}^{l^{\prime}} I_{b}^{j}$. To show that $I_{b}^{(1)}$ is a subcomponent of $I_{b}$ it is sufficient to repeat the argument used in the proof of the finite dimensional case, since if $\overline{B\left(x_{i}, \frac{1}{3} \rho_{i}\right)} \cap \overline{B\left(x_{j}, \frac{1}{3} \rho_{j}\right)} \neq \varnothing$, then either $x_{i} \in B\left(x_{j}, \rho_{j}\right)$ or $x_{j} \in B\left(x_{i}, \rho_{i}\right)$.

Assumption (**). Let $\hat{N}$ be the component of the set $\bigcup_{i=1}^{k+l} \overline{B\left(x_{i}, \frac{1}{3} \rho_{i}\right)}$ which contains the open connected set $N$. Since $h_{1}\left(\tau_{1}^{0}\right), h_{1}\left(\tau_{1}^{1}\right) \in C_{1} \cap \Gamma_{1}^{\prime} \cap I_{b}$ it follows that $h_{1}\left(\tau_{1}^{0}\right), h_{1}\left(\tau_{1}^{1}\right) \in \bigcup_{i=1}^{k+l} \overline{B\left(x_{i}, \frac{1}{3} \rho_{i}\right)}$. There are two cases to be considered, namely $\left\{h_{1}\left(\tau_{1}^{0}\right), h_{1}\left(\tau_{1}^{1}\right)\right\} \subset \hat{N}$ and $\left\{h_{1}\left(\tau_{1}^{0}\right), h_{1}\left(\tau_{1}^{1}\right)\right\} \not \subset \hat{N}$. In the first case it is clear, as in earlier arguments, that $h_{1}\left(\tau_{1}^{0}\right)$ and $h_{1}\left(\tau_{1}^{1}\right)$ are in the same subcomponent of $I_{b}$, and hence in the same component of $I_{b}$.

In the second case, we may assume for instance that $h_{1}\left(\tau_{1}^{0}\right) \notin \hat{N}$. We shall show that $C_{1} \cap \Gamma_{2}^{\prime}=\varnothing$. Otherwise there exists an index $\tilde{s}=1, \ldots, m$ such that $C_{1} \cap \overline{B\left(y_{\tilde{s}}, \frac{2}{3} r_{\tilde{s}}\right)} \neq \varnothing$. Hence, since $C_{1}$ is also connected in $X$, there must be a chain of balls of type

$$
\begin{gathered}
\overline{B\left(x_{i}, \frac{1}{3} \rho_{i}\right)}, \quad i=\frac{1, \ldots, k ;}{\overline{B\left(y_{s}, \frac{2}{3} r_{s}\right)}, \quad \overline{B\left(x_{i}, \frac{1}{3} \rho_{i}\right)}, \quad i=k+1, \ldots, k+l} \quad s=1, \ldots, m
\end{gathered}
$$

joining $h_{1}\left(\tau_{1}^{0}\right)$ and $\overline{B\left(y_{\tilde{s}}, \frac{2}{3} r_{\tilde{s}}\right)}$. Now, repeating the argument already used in the finite dimensional case, mutatis mutandis, we reach the same contradiction. The case $h_{1}\left(\tau_{1}^{1}\right) \notin \hat{N}$ can be treated similarly.

The remaining part of the proof is exactly as in Theorem 1.

4. Further related results. In this section we present two additional theorems whose proofs rely on the main argument introduced in the previous section. The first is a generalization of Theorems 1 and 2, while the second is a result for the case when $K_{b}$ may possibly separate 0 and $e$.

THEOREM 3. Suppose that $X$ is infinite dimensional, or that $X$ is finite dimensional and $K_{b}$ does not separate 0 and e. Assume also that $\overline{M_{b}}$ has only a finite number of components. Then either

$(\alpha) K_{b}$ contains a saddle point of mountain-pass type, or

$(\beta)$ at least one component of $\overline{M_{b}}$ intersects two or more components of $S_{b}$.

PROOF. For simplicity we shall consider only the finite dimensional case, the treatment of the infinite dimensional case being similar. Since the proof requires 
only minor modifications of the argument already given for Theorem 1 we shall indicate only the points of difference. In particular assumption $(* *)$ must be replaced by a more delicate condition.

Let $M_{1}, \ldots, M_{\lambda}, \lambda \geq 1$, be the components of the set $\overline{M_{b}}$. If $(\alpha)$ and $(\beta)$ are assumed false, then either $(*)$ holds or there exists an integer $\lambda^{\prime}$, with $1 \leq \lambda^{\prime} \leq \lambda$, such that

\section{$M_{i}$ intersects exactly one component of $S_{b}$, say $S_{i}$, for every} $i=1, \ldots, \lambda^{\prime}$,

and

$$
M_{i} \cap S_{b}=\varnothing \text { for each } i=\lambda^{\prime}+1, \ldots, \lambda, \text { when } \lambda^{\prime}<\lambda .
$$

Since two different components of $\overline{M_{b}}$ can intersect the same component of $S_{b}$, it is convenient to group the components of $\overline{M_{b}}$ in such a way that

$$
\overline{M_{b}}=\bigcup_{i=1}^{\lambda^{\prime \prime}} \hat{M}_{i}, \quad \lambda^{\prime \prime} \leq \lambda^{\prime},
$$

where $\hat{M}_{i}$ are pairwise disjoint compact sets with the property

(**) each $\hat{M}_{i}, i=1, \ldots, \lambda^{\prime \prime}$, intersects exactly one component $S_{i}$ of $S_{b}$, and $S_{i} \neq S_{j}$ when $i \neq j$.

In this case it is clear that

$$
0<\delta_{0}=\min \left\{\operatorname{dist}\left(\hat{M}_{i} \cup S_{i}, \hat{M}_{j} \cup S_{j}\right): i, j=1, \ldots, \lambda^{\prime \prime}, i \neq j\right\} .
$$

We now introduce an open cover of the compact set $S=\bigcup_{i=1}^{\lambda^{\prime \prime}} S_{i}$. Since $(\alpha)$ is assumed to be false, for each point $x \in S$ we choose an open ball $B\left(x, \rho_{x}\right)$, with radius $\rho_{x}<\delta_{0}$, such that $B\left(x, \rho_{x}\right) \cap I_{b}$ is a subcomponent of $I_{b}$. Let

$$
B\left(x_{1}, \frac{1}{4} \rho_{1}\right), \ldots, B\left(x_{k}, \frac{1}{4} \rho_{k}\right), \quad k \geq 1,
$$

be a finite subcover of the open cover $\left\{B\left(x, \frac{1}{4} \rho_{x}\right): x \in S\right\}$ of $S$, and denote its union by $N$. Also we put $\delta=\min \left\{\delta_{0}, \delta_{1}, \delta_{2}\right\}$.

We now proceed exactly as in the proof of Theorem 1 until the concluding discussion of the assumption $(* *)$.

Assumption $(* *)$. Let $\hat{N}_{i}$ denote the component of $\bigcup_{j=1}^{k+l} B_{j}$ containing the connected set $S_{i}, i=1, \ldots, \lambda^{\prime \prime}$. Since $h_{1}\left(\tau_{1}^{0}\right), h_{1}\left(\tau_{1}^{1}\right) \in C_{1} \cap \Gamma_{1} \cap I_{b}$ it follows that $h_{1}\left(\tau_{1}^{0}\right), h_{1}\left(\tau_{1}^{1}\right) \in \bigcup_{j=1}^{k+l} B_{j}$. There are three cases to consider here:

(a) $\left\{h_{1}\left(\tau_{1}^{0}\right), h_{1}\left(\tau_{1}^{1}\right)\right\} \subset \hat{N}_{i}$ for some $i=1, \ldots, \lambda^{\prime \prime}$;

(b) $\left\{h_{1}\left(\tau_{1}^{0}\right), h_{1}\left(\tau_{1}^{1}\right)\right\} \not \subset \bigcup_{i=1}^{\lambda^{\prime \prime}} \hat{N}_{i}$;

(c) $h_{1}\left(\tau_{1}^{0}\right) \in \hat{N}_{i}$ and $h_{1}\left(\tau_{1}^{1}\right) \in \hat{N}_{k}$ for some $i, k=1, \ldots, \lambda^{\prime \prime}$, with $\hat{N}_{i} \neq \hat{N}_{k}$.

Cases (a) and (b) can be treated exactly as in the proof of Theorem 1. The third case (c) cannot occur, as we now show. Indeed, if $h_{1}\left(\tau_{1}^{0}\right) \in \hat{N}_{i}$ and $h_{1}\left(\tau_{1}^{1}\right) \in \hat{N}_{k}$, then, since $C_{1}$ is connected, there is a chain of closed balls of type (9), (10) or (11) joining $h_{1}\left(\tau_{1}^{0}\right)$ and $h_{1}\left(\tau_{1}^{1}\right)$. For the remaining part of the argument we note that any consecutive set of balls of type (9) or (10) in this chain must belong to the same component of $\bigcup_{j=1}^{k+l} B_{j}$. Now consider the first closed ball $\tilde{B}$ of type (11) in the chain. Since $\delta \leq \delta_{2}$, this ball must be preceded by a closed ball of type (9) 
whose center is in $S_{j} \cap \hat{N}_{i}$ for some $j=1, \ldots, \lambda^{\prime \prime}$. Here, if $j \neq i$ then $\hat{N}_{j}=\hat{N}_{i}$ even though $S_{j} \neq S_{i}$. Since $\delta \leq \delta_{0}$, it is evident that $\tilde{B}$ has center in $\hat{M}_{j}$. The next ball in the chain is either of type (9) or type (11). If it is of type (11) then its center is in $\hat{M}_{j}$ since $\delta \leq \delta_{0}$, while if it is of type (9) its center is in $S_{j}$, again because $\delta \leq \delta_{0}$. In the second case the ball itself is in $\hat{N}_{j}$, and so finally in $\hat{N}_{i}$ since $\hat{N}_{j}=\hat{N}_{i}$. Continuing in this way we see that every closed ball of type (9) or (10) in the chain is contained in $\hat{N}_{i}$. Since $h_{1}\left(\tau_{1}^{1}\right) \in I_{b}$, the last ball in the chain cannot be of type (11) and so is contained in $\hat{N}_{i}$. In particular $h_{1}\left(\tau_{1}^{1}\right) \in \hat{N}_{i}$ and hence $\hat{N}_{i} \cap \hat{N}_{k} \neq \varnothing$, which is absurd.

Now following the remaining part of the proof of Theorem 1, we complete the demonstration.

REMARK. From the proof of Theorem 3 it is easily seen that the assumption concerning $\overline{M_{b}}$ can be replaced by the following:

there exists an integer $\lambda$ such that $\overline{M_{b}}=\bigcup_{i=1}^{\lambda} M_{j}$ where $M_{j}, j=$ $1, \ldots, \lambda$, are pairwise disjoint compact sets.

The appropriate assertion $(\beta)$ then becomes

$$
\begin{aligned}
& \text { there exists at least one set } M_{j}, j=1, \ldots, \lambda \text {, which intersects two } \\
& \text { or more components of } S_{b} \text {. }
\end{aligned}
$$

This version of Theorem 3 contains Theorem 1 when $\lambda=1$, and reduces exactly to Theorem 3 if each set $M_{j}, j=1, \ldots, \lambda$, is connected.

In the remaining part of this section we shall prove a further result without invoking the hypothesis that $K_{b}$ does not separate the points 0 and $e$.

THEOREM 4. The critical set $K_{b}$ in the Mountain Pass Theorem is such that either

$(\alpha) K_{b}$ contains at least one point of mountain-pass type, or

( $\beta) \overline{M_{b}}$ intersects at least two components of $S_{b} \cup P_{b}$.

PROOF. We shall present the demonstration only for the finite dimensional case. Thus without loss of generality we may assume that $X$ is endowed with the Euclidean norm. Also for convenience in notation we shall put $S_{b}^{*}=S_{b} \cup P_{b}$.

We start by assuming that there are no points in $S_{b}^{*}$ of m-p type, for otherwise $(\alpha)$ holds and we are done. Thus we suppose for contradiction that either

$$
\overline{M_{b}} \cap S_{b}^{*}=\varnothing
$$

or

$$
\overline{M_{b}} \text { intersects exactly one component, say } S \text {, of } S_{b}^{*} \text {. }
$$

Under assumption (*) we have $0<\delta=\operatorname{dist}\left(\overline{M_{b}}, S_{b}^{*}\right)$, whenever the sets $M_{b}$ and $S_{b}^{*}$ are nonempty. If, on the other hand, one or the other of these sets is empty, then we put $\delta=1$.

Next suppose $(* *)$ holds. Since $(\alpha)$ is assumed to be false, for each point $x \in S$ we can choose an open ball $B\left(x, \rho_{x}\right)$ such that the set $B\left(x, \rho_{x}\right) \cap I_{b}$ is a subcomponent of $I_{b}$, which is nonempty since $x$ is either a saddle point or a proper local maximum. 
Hence, the open cover $\left\{B\left(x, \frac{1}{4} \rho_{x}\right): x \in S\right\}$ of the compact set $S$ possesses a finite subcover, namely

$$
B\left(x_{1}, \frac{1}{4} \rho_{1}\right), \ldots, B\left(x_{k}, \frac{1}{4} \rho_{k}\right), \quad k \geq 1,
$$

whose union will be denoted by $N$. By virtue of assumption (**) the open set $N$ is also connected.

We have $0<\delta=\operatorname{dist}\left(\overline{M_{b}}, S_{b}^{*} \cap N^{c}\right)$, if $S_{b}^{*} \cap N^{c} \neq \varnothing$. When $S_{b}^{*} \cap N^{c}=\varnothing$ we take $\delta=1$, as before.

For uniformity of notation we put $N=\varnothing$ and $k=0$ also for the simpler case (*). In both cases, we denote the set $K_{b} \cap N^{c}$ by $\hat{K}_{b}$.

For each point $x \in \hat{K}_{b} \cap S_{b}^{*}$ we can select an open ball $B\left(x, \rho_{x}\right)$ with radius $\rho_{x}<\delta$ such that the set $B\left(x, \rho_{x}\right) \cap I_{b}$ is a subcomponent of $I_{b}$. If $y \in \hat{K}_{b} \cap M_{b}$ we choose an open ball $B\left(y, r_{y}\right)$ such that $I \geq b$ in $B\left(y, r_{y}\right)$. From the open cover $\left\{B\left(x, \frac{1}{4} \rho_{x}\right), B\left(y, \frac{1}{2} r_{y}\right): x \in \hat{K}_{b} \cap S_{b}^{*}, y \in \hat{K}_{b} \cap M_{b}\right\}$ of the compact set $\hat{K}_{b}$ we can extract a finite subcover of the form

$$
\begin{gathered}
B\left(x_{k+1}, \frac{1}{4} \rho_{k+1}\right), \ldots, B\left(x_{k+l}, \frac{1}{4} \rho_{k+l}\right), \\
B\left(y_{1}, \frac{1}{2} r_{1}\right), \ldots, B\left(y_{m}, \frac{1}{2} r_{m}\right),
\end{gathered}
$$

whose union will be denoted by $W$.

In the following, $U$ will denote the open neighborhood $N \cup W$ of $K_{b}$. Since $U$ is given by a finite union of open balls, its components $U_{j}, j=1, \ldots, \mu, \mu \leq 1+l+m$, which are of course open, can be supposed to have the additional property that

$$
0<\min \left\{\operatorname{dist}\left(\overline{U_{i}}, \overline{U_{j}}\right): i, j=1, \ldots, \mu, i \neq j\right\} .
$$

This can be done by slightly reducing the numbers $\rho_{i}, i=k+1, \ldots, k+l$, and $r_{s}$, $s=1, \ldots, m$.

We now proceed exactly as in the proof of Theorem 1 until formula (17).

The next step is to prove that there exists a bridge $v_{j}$ corresponding to each pair of points $z_{j}^{0}$ and $z_{j}^{1}, j=1, \ldots, \nu$. We start by constructing the bridge $v_{1}$ from $z_{1}^{0}$ to $z_{1}^{1}$. We recall that $z_{1}^{0}$ and $z_{1}^{1}$ are both in $I_{b}$ by (16). For convenience we discuss assumptions $(*)$ and $(* *)$ separately.

Assumption $(*)$. Here $k=0$ and $\overline{M_{b}} \cap S_{b}^{*}=\varnothing$. Thus $U_{1}$ consists entirely of balls of type (25), since it is connected and the radii $\rho_{i}, r_{s}, i=1, \ldots, l, s=1, \ldots, m$, are each less than $\delta$. Moreover $\overline{U_{1}} \cap I_{b}=I_{b}^{(1)}$ is a subcomponent of $I_{b}$ by virtue of the argument already used to treat the corresponding assumption $(*)$ in the proof of Theorem 1 . Hence there exists a bridge $v_{1}$ joining $z_{1}^{0}$ and $z_{1}^{1}$, as required.

Assumption (**). Let $\hat{N}$ be the component of the set $\bigcup_{i=1}^{k+l} B_{i}$ which contains the open connected set $N$. There are two cases to consider: $\left\{z_{1}^{0}, z_{1}^{1}\right\} \subset \hat{N}$ and $\left\{z_{1}^{0}, z_{1}^{1}\right\} \not \subset \hat{N}$. In the first case $z_{1}^{0}, z_{1}^{1} \in \hat{N} \cap I_{b}$, where $\hat{N} \cap I_{b}=I_{b}^{(1)}$ is a subcomponent of $I_{b}$ by construction. Consequently there is a bridge $v_{1}$ joining $z_{1}^{0}$ and $z_{1}^{1}$.

Otherwise we may assume, for instance, that $z_{1}^{0} \notin \hat{N}$. In this case we claim that $U_{1}$ consists entirely of balls of type (25). Indeed, if there were some ball of type (26) in $U_{1}$, then there would be a chain consisting of closed balls of type (24) and (25) joining $z_{1}^{0}$ to a closed ball of type (26). By definition of $\hat{N}$ and the fact that $z_{1}^{0} \notin \hat{N}$, however, no balls of type (24) can appear in the chain. Moreover, closed 
balls of type (25) are disjoint from closed balls of type (26). Hence no balls of type (26) can appear in $U_{1}$ and, by the same argument repeated again, also no balls of type (24). The situation is thus reduced to the case already discussed under assumption $(*)$ and so a bridge $v_{1}$ joining $z_{1}^{0}$ to $z_{1}^{1}$ can be found. The case $z_{1}^{1} \notin \hat{N}$ can be handled similarly.

For each $j=2, \ldots, \nu$ we proceed as above to find a bridge $v_{j}$ for the component $U_{j}$.

Finally, denoting by $\tilde{g}$ the path obtained by following, first, $g$ from $0=z_{0}^{1}$ to $z_{1}^{0}$, then $v_{1}$ from $z_{1}^{0}$ to $z_{1}^{1}$, next $g$ from $z_{1}^{1}$ to $z_{2}^{0}$ and so forth until reaching $e=z_{\nu+1}^{0}$, we obtain the required contradiction.

REMARK. In analogy with Theorem 3, it is clear that Theorem 4 can be generalized as follows:

Assume that $\overline{M_{b}}=\bigcup_{j=1}^{\lambda} M_{j}$, where $M_{j}, j=1, \ldots, \lambda$, are pairwise disjoint compact sets. Then either

$(\alpha) K_{b}$ contains a point of mountain-pass type, or

$\left(\beta^{\prime}\right)$ at least one set $M_{j}$ intersects two or more components of $S_{b} \cup P_{b}$.

ACKNOWLEDGMENT. The work of the first author was carried out under the auspices of the Gruppo Nazionale di Analisi Funzionale e sue Applicazioni of the Consiglio Nazionale delle Richerche, Rome, Italy. The paper was written partly at the University of Perugia and partly at the University of Minnesota. The first author wishes to thank the University of Minnesota for its hospitality, and the second author similarly expresses his thanks to the University of Perugia for its support.

We wish finally to thank Professor Helmut Hofer for several helpful comments concerning this work.

\section{REFERENCES}

1. A. Ambrosetti and P. H. Rabinowitz, Dual variational methods in critical point theory and applications, J. Funct. Anal. 14 (1973), 349-381.

2. D. C. Clark, A variant of the Lusternik-Schnirelman theory, Indiana Univ. Math. J. 22 (1972), 65-74.

3. I. Ekeland and H. Hofer, Periodic solutions with prescribed minimal period for convex autonomous hamiltonian systems, Invent. Math. 81 (1985), 155-188.

4. H. Hofer, A geometric description of the neighbourhood of a critical point given by the Mountain Pass Theorem, J. London Math. Soc. 31 (1985), 566-570.

5. L. Nirenberg, Variational and topological methods in nonlinear problems, Bull. Amer. Math. Soc. (N.S.) 4 (1981), 267-302.

6. P. Pucci and J. Serrin, A Mountain Pass Theorem, J. Differential Equations 60 (1985), 142-149.

7. __ Extensions of the Mountain Pass Theorem, J. Funct. Anal. 59 (1984), 185-210.

8. P. H. Rabinowitz, Variational methods for nonlinear eigenvalue problems, Eigenvalues of Nonlinear Problems (G. Prodi, ed.), C.I.M.E., Edizioni Cremonese, Rome, 1974, pp. 141-195.

9. 1983.

Dipartimento di Matematica, Università Degli Studi, 06100 Perugia, Italy

Department of Mathematics, University of Minnesota, Minneapolis, MINNESOTA 